\title{
APPROXIMATION FROM LOCALLY FINITE-DIMENSIONAL SHIFT-INVARIANT SPACES
}

\author{
KANG ZHAO
}

(Communicated by J. Marshall Ash)

\begin{abstract}
After exploring some topological properties of locally finitedimensional shift-invariant subspaces $S$ of $L_{p}\left(\mathbb{R}^{s}\right)$, we show that if $S$ provides approximation order $k$, then it provides the corresponding simultaneous approximation order. In the case $S$ is generated by a compactly supported function in $L_{\infty}(\mathbb{R})$, it is proved that $S$ provides approximation order $k$ in the $L_{p}(\mathbb{R})$-norm with $p>1$ if and only if the generator is a derivative of a compactly supported function that satisfies the Strang-Fix conditions.
\end{abstract}

\section{INTRODUCTION}

Let $S$ be a linear space consisting of functions defined on $\mathbb{R}^{s} . S$ is said to be shift invariant if $f(\cdot+\alpha)$ lies in $S$ whenever $f$ does, for every $\alpha \in \mathbb{Z}^{s} . S$ is said to be locally finite-dimensional if the restriction of $S$ to any bounded subset of $\mathbb{R}^{s}$ is finite-dimensional. A typical example for such spaces is the linear span $S$ of a finite number of compactly supported functions and their shifts. That is,

$$
S=S_{0}(\Phi):=\operatorname{span}\left\{\varphi(\cdot+\alpha): \alpha \in \mathbb{Z}^{s}, \varphi \in \Phi\right\},
$$

with $\Phi$ a finite family of compactly supported functions. When $\Phi$ consists of one function $\varphi$, we denote $S_{0}(\Phi)$ by $S_{0}(\varphi)$. $S_{0}(\Phi)$ is usually called a finitely generated shift-invariant space. It is clear that the shift-invariance and the local finitedimension are purely algebraic properties. In this paper we shall show how to probe the (simultaneous) approximation order provided by $S$ by means of these two algebraic properties.

Let $m \geq 0$ be an integer and $k>0$. A subspace $S$ of $L_{p}\left(\mathbb{R}^{s}\right)$ is said to provide simultaneous approximation order $(m, k)$ if

$$
\inf _{g \in S} \sum_{j=0}^{m} \sum_{|\alpha|=j} h^{j}\left\|D^{\alpha}(f-g(\cdot / h))\right\|_{p} \leq C_{f} h^{k}
$$

as $h \rightarrow 0+$, for every $f \in W_{p}^{m}\left(\mathbb{R}^{s}\right) \cap W_{p}^{k}\left(\mathbb{R}^{s}\right)$. Here, $C_{f}$ is a constant independent of $h$ and $D^{\alpha}$ is the $\alpha$-order differentiation operator. By convention, $S$ is said to provide approximation order $k$ when it provides simultaneous approximation order $(0, k)$. The simultaneous approximation order of shift-invariant subspaces generated by a

Received by the editors June 28, 1994 and, in revised form, December 13, 1994.

1991 Mathematics Subject Classification. Primary 41A15, 41A25, 41A28, 41A63.

Key words and phrases. Approximation order, locally finite-dimensional, polynomial reproducing, shift-invariant space, simultaneous approximation, Strang-Fix condition. 
finite number of functions has been of interest in Approximation Theory and Finite Element Analysis for a long time and it is well known [1], [11], [12] that $S_{0}(\varphi)$ provides approximation order $k$ if $\varphi \in L_{p}\left(\mathbb{R}^{s}\right)$ with $p \geq 1$ is compactly supported and satisfies the so-called Strang-Fix conditions of order $k$ :

(i) $\hat{\varphi}(0) \neq 0$;

(ii) $D^{\alpha} \hat{\varphi}=0$ on $2 \pi \mathbb{Z}^{s} \backslash 0$, for all $|\alpha|<k$.

Here, $\hat{\varphi}$ denotes the Fourier transform of $\varphi$. The Strang-Fix conditions have been so well reputed because they enable us to determine the approximation order provided by $S_{0}(\varphi)$, with $\hat{\varphi}(0) \neq 0$, by examining the single generator $\varphi$, in spite of the fact that $S_{0}(\varphi)$ is infinite-dimensional.

It is well known that the above-mentioned Strang-Fix conditions can also be described algebraically as follows. Denote by $\varphi^{* \prime}$ the discrete convolution mapping with $\varphi$. Namely,

$$
\varphi^{* \prime}: f \rightarrow \sum_{\alpha \in \mathbb{Z}^{s}} \varphi(\cdot-\alpha) f(\alpha) .
$$

Since $\varphi$ is assumed to be compactly supported, for each $x \in \mathbb{R}^{s}, \varphi^{* \prime} f(x)$ is a sum of finite number of terms. As shown in his paper [11], Shoenberg observed that $S_{0}(\varphi)$ provides approximation order $k$ if $\varphi^{* \prime}$ maps $\Pi_{k-1}$ onto $\Pi_{k-1}$ in the univariate case, where $\Pi_{k-1}$ is the linear space of all polynomials of degree $<k$. As is well known now, the Strang-Fix conditions of order $k$ are equivalent to that $\varphi^{* \prime}$ maps $\Pi_{k-1}$ onto $\Pi_{k-1}$. Therefore, $S_{0}(\varphi)$ provides approximation order $k$ if $\varphi$ has the algebraic property that $\varphi^{* \prime}$ maps $\Pi_{k-1}$ onto $\Pi_{k-1}$. In his recent paper [8], Jia has shown the following interesting fact: For any function $f$ defined on $\mathbb{R}^{s}$, there exists $a: \mathbb{Z}^{s} \rightarrow \mathbb{C}^{s}$ such that $f=\varphi^{* \prime} a$ if and only if $S_{0}(\varphi)$ locally contains $f$. Here, $S_{0}(\varphi)$ is said to locally contain $f$ if the restriction of $S_{0}(\varphi)$ to any compact subset $B$ contains the restriction of $f$ to $B$. Therefore, if $\hat{\varphi}(0) \neq 0$, then $S_{0}(\varphi)$ provides approximation order $k$ if and only if $S_{0}(\varphi)$ locally contains $\Pi_{k-1}[8]$. We note that $S_{0}(\varphi)$ locally containing $\Pi_{k-1}$ cannot guarantee it to provide approximation order $k$ in general. When $\hat{\varphi}(0) \neq 0$, it is well known that there is a local approximation scheme that realizes the approximation order provided by $S_{0}(\varphi)[1]$.

The condition that $\hat{\varphi}(0) \neq 0$ has been assumed in the past study of approximation order of $S_{0}(\varphi)$. As shown by Strang and Fix [12], for any compactly supported $\varphi \in L_{2}\left(\mathbb{R}^{s}\right)$, if $S_{0}(\varphi)$ provides an approximation order $k$ via a controlled approximation scheme, then this condition is also necessary. But $S_{0}(\varphi)$ may provide a positive approximation order even if $\hat{\varphi}(0)=0$. One well-known example is the function

$$
\varphi(x)= \begin{cases}1, & \text { if } 0 \leq x \leq 1 \\ -1, & \text { if } 1<x \leq 2 \\ 0, & \text { else }\end{cases}
$$

It is known that $S_{0}(\varphi)$ indeed provides approximation order 1 in the $L_{2}(\mathbb{R})$-norm. In the recent paper by de Boor, DeVore, and Ron [4], the authors obtained a necessary and sufficient condition for $S_{0}(\varphi)$ to provide approximation order $k$ in the $L_{2}\left(\mathbb{R}^{s}\right)$-norm, for any generator $\varphi \in L_{2}\left(\mathbb{R}^{s}\right)$. A corresponding result of a necessary and sufficient condition under which $S_{0}(\varphi)$ provides simultaneous approximation order $(m, k)$ has been presented in [13], for any $\varphi \in W_{2}^{m}\left(\mathbb{R}^{s}\right)$. As shown in [13], for 
any compactly supported univariate function $\varphi \in W_{2}^{m}(\mathbb{R}), S_{0}(\varphi)$ provides simultaneous approximation order $(m, k)$ in the $L_{2}(\mathbb{R})$-norm if and only if there exist a neighborhood $\Omega_{\alpha}$ of the origin and a constant $C_{\alpha}$ such that

$$
|\hat{\varphi}(x+2 \pi \alpha)| \leq C_{\alpha}|x|^{k}|\hat{\varphi}(x)|, \quad \forall x \in \Omega_{\alpha},
$$

for all $\alpha \in \mathbb{Z} \backslash 0$. In particular, this implies that if $S_{0}(\varphi)$ provides approximation order $k$, then it also provides simultaneous approximation order $(m, k)$.

In the next sections we shall prove that, for any shift-invariant subspace $S \subset$ $W_{p}^{m}\left(\mathbb{R}^{s}\right)$ that is locally finite-dimensional, if $S$ provides approximation order $k$, then it also provides simultaneous approximation order $(m, k)$. In the univariate case, we shall prove that, for any nontrivial compactly supported $\varphi \in L_{p}(\mathbb{R}) \cap L_{p^{\prime}}(\mathbb{R})$ with $p>1, S_{0}(\varphi)$ provides approximation order $k$ if and only if $\varphi$ satisfies (1.3) for all $\alpha \in \mathbb{Z} \backslash 0$, where $p^{\prime}=p /(p-1)$ is the conjugate number of $p$. In other words, in this case, $S_{0}(\varphi)$ provides approximation order $k$ if and only if

$$
D^{\alpha} \hat{\varphi}=0 \quad \text { on } 2 \pi \mathbb{Z} \backslash 0, \forall 0 \leq \alpha<k+m,
$$

where $m$ is the smallest integer such that $D^{m} \hat{\varphi}(0) \neq 0$. As we shall see, any compactly supported function $\varphi \in L_{p}(\mathbb{R})$ satisfies (1.4) if and only if it is the $m$ thorder derivative of a compactly supported function that satisfies the Strang-Fix condition of order $k+m$, with $m$ the smallest integer such that $D^{m} \hat{\varphi}(0) \neq 0$. Therefore, among the compactly supported functions in $L_{p}(\mathbb{R})$ are only those that satisfy the Strang-Fix conditions or their derivatives the candidates for generating a shift-invariant space that may provide a positive approximation order.

\section{LOCALLY FINITE-DIMENSIONAL SHIFT-INVARIANT SPACES}

In this section we show some nice topological properties owned by every shiftinvariant subspace $S \subset L_{p}\left(\mathbb{R}^{s}\right)$ that is locally finite-dimensional.

Proposition 2.1. Let $S \subset L_{p}\left(\mathbb{R}^{s}\right)$ be a shift-invariant subspace with $1 \leq p \leq \infty$ such that $S$ is locally finite-dimensional. For any linear mapping $A: S \rightarrow L_{q}\left(\mathbb{R}^{s}\right)$, with $1 \leq q \leq \infty$, if $A$ commutes with integer translations, then there exist two positive constants $C_{1}$ and $C_{2}$ such that, for all $f \in S$,

$$
\begin{aligned}
& C_{1}\left(\sum_{\alpha \in \mathbb{Z}^{s}} \min _{g \in \operatorname{ker} A_{\mid}}\|f(\cdot+\alpha)-g\|_{L_{p}\left([0 . .1]^{s}\right)}^{q}\right)^{1 / q} \\
& \quad \leq\|A f\|_{q} \leq C_{2}\left(\sum_{\alpha \in \mathbb{Z}^{s}} \min _{g \in \operatorname{ker} A_{\mid}}\|f(\cdot+\alpha)-g\|_{L_{p}\left([0 . .1]^{s}\right)}^{q}\right)^{1 / q}
\end{aligned}
$$

when $q<\infty$,

$$
\begin{aligned}
& C_{1} \sup _{\alpha \in \mathbb{Z}^{s}} \min _{g \in \operatorname{ker} A_{\mid}}\|f(\cdot+\alpha)-g\|_{L_{p}\left([0 . .1]^{s}\right)} \\
& \quad \leq\|A f\|_{q} \leq C_{2} \sup _{\alpha \in \mathbb{Z}^{s}} \min _{g \in \operatorname{ker} A_{\mid}}\|f(\cdot+\alpha)-g\|_{L_{p}\left([0 . .1]^{s}\right)}
\end{aligned}
$$

when $q=\infty$, where $\operatorname{ker} A_{\mid}:=\left\{f \in S: A f=0\right.$ on $\left.[0 . .1]^{s}\right\}$.

Proof. It is clear that $\operatorname{ker} A_{\mid}$is a subspace of $S$. For any $f \in S$ and $g \in S$, the restriction of $A f$ to $[0 . .1]^{s}$ equals the restriction of $A g$ to $[0 . .1]^{s}$ if and only if

$$
A(f-g)=A f-A g=0 \quad \text { on }[0 . .1]^{s} \text {. }
$$


This proves that the restriction of the range of $A$ to $[0 . .1]^{s}$ is isomorphic to the restriction of the quotient space $S / \operatorname{ker} A_{\mid}$to $[0 . .1]^{s}$. Since $S$ is locally finitedimensional, the restrictions of $S / \operatorname{ker} A$ and $S$ to $[0 . .1]^{s}$ are finite-dimensional. As is well known, any linear mapping on a finite-dimensional normed space is bounded and any two norms on a finite-dimensional space are equivalent. Consequently, there exist two positive constants $C_{1}$ and $C_{2}$ such that

$$
C_{1} \min _{g \in \operatorname{ker} A_{\mid}}\|f-g\|_{L_{p}\left([0 . .1]^{s}\right)} \leq\|A f\|_{L_{q}\left([0 . .1]^{s}\right)} \leq C_{2} \min _{g \in \operatorname{ker} A_{\mid}}\|f-g\|_{L_{p}\left([0 . .1]^{s}\right)}
$$

for all $f \in S$. Since $S$ is shift invariant, $f \in S$ implies that $f(\cdot+\alpha) \in S$ for any $\alpha \in \mathbb{Z}^{s}$. Also we have that

$$
(A f)(\cdot+\alpha)=A(f(\cdot+\alpha)), \quad \forall \alpha \in \mathbb{Z}^{s}
$$

because $A$ commutes with integer translations. When $q=\infty,(2.2)$ follows from (2.3) and the fact that $S$ is shift invariant. When $q<\infty$, it follows from (2.3) that, for any $f \in S$,

$$
\begin{aligned}
\int_{\mathbb{R}^{s}}|(A f)(x)|^{q} d x & =\sum_{\alpha \in \mathbb{Z}^{s}} \int_{[0 . .1]^{s}}|(A f)(x+\alpha)|^{q} d x \\
& =\sum_{\alpha \in \mathbb{Z}^{s}} \int_{[0 . .1]^{s}}|A(f(x+\alpha))|^{q} d x \\
& \leq \sum_{\alpha \in \mathbb{Z}^{s}} C_{2}^{q} \min _{g \in \operatorname{ker} A_{\mid}}\left(\int_{[0 . .1]^{s}}|f(x+\alpha)-g|^{p} d x\right)^{q / p} \\
& =C_{2}^{q} \sum_{\alpha \in \mathbb{Z}^{s}} \min _{g \in \operatorname{ker} A_{\mid}}\|f(\cdot+\alpha)-g\|_{L_{p}\left([0 . .1]^{s}\right)^{.}}^{q}
\end{aligned}
$$

Analogously we can establish the other inequality of (2.1).

In the case where $p=q$ we obtain that $\|A f\|_{p} \leq C_{2}\|f\|_{p}$.

Corollary 2.2. Let $1 \leq p \leq \infty$ and $S \subset L_{p}\left(\mathbb{R}^{s}\right)$ be a shift-invariant subspace that is locally finite-dimensional. If $A: S \rightarrow L_{p}\left(\mathbb{R}^{s}\right)$ is a linear mapping that commutes with integer translations, then $A$ is bounded.

For any polynomial of degree $n: p_{n}=\sum_{|\alpha| \leq n} a_{\alpha}(\cdot)^{\alpha}$, by $p_{n}(D)$ we mean the differential operator

$$
p_{n}(D):=\sum_{|\alpha| \leq n} a_{\alpha} D^{\alpha} .
$$

It is clear that $p_{n}(D)$ is a linear mapping from $W_{p}^{n}\left(\mathbb{R}^{s}\right)$ to $L_{p}\left(\mathbb{R}^{s}\right)$. For any sufficiently smooth function $f$ and any $t \in \mathbb{R}^{s}$,

$$
D^{\alpha}(f(\cdot+t))=\left(D^{\alpha} f\right)(\cdot+t) .
$$

This shows that $p_{n}(D)$ commutes with integer translations.

Corollary 2.3. Let $p_{n}$ be any polynomial in s-variable of degree $n$ and $S$ a shiftinvariant subspace of $W_{p}^{n}\left(\mathbb{R}^{s}\right)$ that is locally finite-dimensional. Then there exists a constant $C$ such that $\left\|p_{n}(D) f\right\|_{p} \leq C\|f\|_{p}$ for all $f$ in $S$. 
It is well known that

$$
\left(\sum_{\alpha \in \mathbb{Z}^{s}}|a(\alpha)|^{q}\right)^{1 / q} \leq\left(\sum_{a \in \mathbb{Z}^{s}}|a(\alpha)|^{p}\right)^{1 / p}
$$

if $1 \leq p \leq q \leq \infty$ and $a \in l_{q}\left(\mathbb{Z}^{s}\right)$. From the proof of Proposition 2.1 we obtain

Corollary 2.4. Let $1 \leq p<q \leq \infty$ and $S \subset L_{p}\left(\mathbb{R}^{s}\right)$ be a shift-invariant subspace that is locally finite-dimensional and is locally contained in $L_{q}\left(\mathbb{R}^{s}\right)$. Then, $S \subset$ $L_{q}\left(\mathbb{R}^{s}\right)$ and there exists a constant $C$ such that $\|f\|_{q} \leq C\|f\|_{p}$ for all $f \in s$.

Corollary 2.5. Let $S \subset L_{p}\left(\mathbb{R}^{s}\right) \cap L_{q}\left(\mathbb{R}^{s}\right)$ be a shift-invariant subspace that is locally finite-dimensional, with $1 \leq p<q \leq \infty$. Then the closure of $S$ in $L_{p}\left(\mathbb{R}^{s}\right)$ is contained in the closure of $S$ in $L_{q}\left(\mathbb{R}^{s}\right)$.

When $S=S_{0}(\Phi)$ is generated by a finite number of compactly supported functions in $L_{p}\left(\mathbb{R}^{s}\right)$, we denote by $S_{*}(\Phi)$ the closure of $S_{0}(\Phi)$ in the topology of pointwise convergence. Namely, $f \in S_{*}(\Phi)$ if and only if there is a sequence $s_{j} \in S_{0}(\Phi)$ such that $\lim _{j \rightarrow \infty} s_{j}(x)=f(x)$ for almost every $x \in \mathbb{R}^{s}$. One can verify that $S_{*}(\Phi)$ consists of all functions of the form

$$
\sum_{\varphi \in \Phi} \varphi^{* \prime} a_{\varphi}
$$

for all $a_{\varphi}: \mathbb{Z}^{s} \rightarrow \mathbb{C}^{s}$. As shown in [8], $f$ is contained in $S_{*}(\Phi)$ if and only if $f$ is locally contained in $S_{0}(\Phi)$. Since the topology of $L_{p}\left(\mathbb{R}^{s}\right)$ is stronger than that of $S_{*}(\Phi)$, we have the following corollary that was first observed by Jia.

Corollary 2.6. For any finite family $\Phi$ of compactly supported functions in $L_{p}\left(\mathbb{R}^{s}\right)$, $S_{*}(\Phi) \cap L_{p}\left(\mathbb{R}^{s}\right)$ is a closed subspace of $L_{p}\left(\mathbb{R}^{s}\right)$.

Since $S_{*}(\Phi) \cap L_{p}\left(\mathbb{R}^{s}\right)$ is also shift-invariant and locally finite-dimensional, from Corollary 2.4 we obtain

Proposition 2.7. For any $1 \leq p<q \leq \infty$ and any finite $\Phi \subset L_{p}\left(\mathbb{R}^{s}\right) \cap L_{q}\left(\mathbb{R}^{s}\right)$ consisting of compactly supported functions, we have that $S_{*}(\Phi) \cap L_{p}\left(\mathbb{R}^{s}\right) \subset S_{*}(\Phi) \cap$ $L_{q}\left(\mathbb{R}^{s}\right)$.

For $S_{0}(\Phi) \subset L_{p}\left(\mathbb{R}^{s}\right)$, denote by $S_{p}(\Phi)$ the closure of $S_{0}(\Phi)$ in $L_{p}\left(\mathbb{R}^{s}\right)$. Let $\varphi$ be a compactly supported function in $L_{2}\left(\mathbb{R}^{s}\right)$. As proved by de Boor, DeVore, Ron [4], $S_{*}(\varphi) \cap L_{2}\left(\mathbb{R}^{s}\right)$ is a subspace of $S_{2}(\varphi)$. Since $S_{*}(\varphi) \cap L_{2}\left(\mathbb{R}^{s}\right)$ contains $S_{0}(\varphi)$ and is closed, it follows that $S_{2}(\varphi)=S_{*}(\varphi) \cap L_{2}\left(\mathbb{R}^{s}\right)$. We shall show that this has an extension to the case where $1<p<2$ and $\varphi \in L_{p^{\prime}}\left(\mathbb{R}^{s}\right)$. Recall that $p^{\prime}=p /(p-1)$. For the proof we need

Proposition 2.8. Let $1<p<\infty$ and $\Phi \subset L_{p}\left(\mathbb{R}^{s}\right) \cap L_{p^{\prime}}\left(\mathbb{R}^{s}\right)$ be a finite family of compactly supported functions. Then, $S_{p^{\prime}}(\Phi)$ can be identified with the dual space $\left(S_{p}(\Phi)\right)^{*}$ of $S_{p}(\Phi)$

Proof. As $S_{p}(\Phi)$ is a closed subspace of $L_{p}\left(\mathbb{R}^{s}\right)$, we know that the dual space of $S_{p}(\Phi)$ is isomorphic to the quotient space $L_{p^{\prime}}\left(\mathbb{R}^{s}\right) /\left(S_{p}(\Phi)\right)^{\perp}[10]$. Since $L_{p}\left(\mathbb{R}^{s}\right)$ and $L_{p^{\prime}}\left(\mathbb{R}^{s}\right)$ are reflexive and $S_{p}(\Phi)$ is a closed subspace of $L_{p}\left(\mathbb{R}^{s}\right)$, we have that

$$
\left(S_{p}(\Phi)\right)^{* *}=\left(L_{p^{\prime}}\left(\mathbb{R}^{s}\right) /\left(S_{p}(\Phi)\right)^{\perp}\right)^{*}=\left(S_{p}(\Phi)\right)^{\perp \perp}=S_{p}(\Phi) .
$$

It suffices to prove the case where $p \leq 2$ because, otherwise, $p^{\prime} \leq 2$ and $\left(S_{p^{\prime}}(\Phi)\right)^{*}=$ $\left(S_{p}(\Phi)\right)^{* *}=S_{p}(\Phi)$. First we prove that $S_{p^{\prime}}(\Phi)$ is dense in $\left(S_{p}(\Phi)\right)^{*}$. For any 
$f \in\left(S_{p}(\Phi)\right)^{* *}=S_{p}(\Phi)$ that annihilates $S_{p^{\prime}}(\Phi), \int_{\mathbb{R}^{s}}|f|^{2}=0$ because $f$ lines in $S_{p}(\Phi) \subset S_{p^{\prime}}(\Phi)$. So $f=0$. This proves that $S_{p^{\prime}}(\Phi)$ is dense in $\left(S_{p}(\Phi)\right)^{*}$. Therefore, $S_{p}(\Phi)=\left(S_{p}(\Phi)\right)^{* *}=\left(S_{p^{\prime}}(\Phi)\right)^{*}$ and

$$
S_{p^{\prime}}(\Phi)=\left(S_{p^{\prime}}(\Phi)\right)^{* *}=\left(S_{p}(\Phi)\right)^{*} .
$$

Theorem 2.9. Let $1<p \leq 2$ and $p^{\prime}=p /(p-1)$. For any compactly supported $\varphi \in L_{p^{\prime}}\left(\mathbb{R}^{s}\right), S_{p}(\varphi)=S_{*}(\varphi) \cap L_{p}\left(\mathbb{R}^{s}\right)$.

Proof. By Corollary 2.5 and Proposition 2.7,

$$
S_{p}(\varphi) \subset S_{*}(\varphi) \cap L_{p}\left(\mathbb{R}^{s}\right) \subset S_{*}(\varphi) \cap L_{2}\left(\mathbb{R}^{s}\right)=S_{2}(\varphi) \subset S_{p^{\prime}}(\varphi) .
$$

This implies the dual space of $S_{*}(\varphi) \cap L_{p}\left(\mathbb{R}^{s}\right)$ contains that of $S_{p}(\varphi)$. As we know, $S_{p}(\varphi)$ and $S_{*}(\varphi) \cap L_{p}\left(\mathbb{R}^{s}\right)$ both are closed in $L_{p}\left(\mathbb{R}^{s}\right)$. From the reflexivity it follows that some closed subspace of $S_{p^{\prime}}(\varphi)$ can be identified with $\left(S_{*}(\varphi) \cap L_{p}\left(\mathbb{R}^{s}\right)\right)^{*}$. From Proposition 2.8 we know that $S_{p^{\prime}}(\varphi)$ can be identified with the dual space of $S_{p}(\varphi)$. Hence, $S_{p}(\varphi)$ and $S_{*}(\varphi) \cap L_{p}\left(\mathbb{R}^{s}\right)$ have the same dual space $S_{p^{\prime}}(\varphi)$. Thus we obtain that

$$
S_{p}(\varphi)=\left(S_{p}(\varphi)\right)^{* *}=\left(S_{*}(\varphi) \cap L_{p}\left(\mathbb{R}^{s}\right)\right)^{* *}=S_{*}(\varphi) \cap L_{P}\left(\mathbb{R}^{s}\right)
$$

\section{Application 1: Multivariate approximation}

In this section we apply the results obtained in Section 2 to obtain some results about multivariate approximation from shift-invariant spaces that are locally finitedimensional.

Theorem 3.1. Let $S \subset W_{p}^{m}\left(\mathbb{R}^{s}\right)$ be a shift-invariant subspace that is locally finitedimensional. If $S$ provides approximation order $k$ in the $L_{p}\left(\mathbb{R}^{s}\right)$-norm, then $S$ also provides simultaneous approximation order $(m, k)$.

Proof. For any $f \in W_{p}^{m}\left(\mathbb{R}^{s}\right)$, there exists $s_{h} \in S$ such that

$$
\left\|f-s_{h}(\cdot / h)\right\|_{p} \leq C_{f} h^{k}
$$

with some constant $C_{f}$ independent of $h$. Let $\psi$ be any compactly supported function in $W_{p}^{m}\left(\mathbb{R}^{s}\right)$ such that $S_{0}(\psi)$ provides simultaneous approximation order $(m, k)$. For instance, we can choose $\psi$ as a tensor product of some univariate B-spline functions [5]. Let

$$
S_{+}:=S+S_{0}(\psi) .
$$

That is, $S_{+}$is the space spanned by $S$ and $S_{0}(\psi)$. It is clear that $S_{+}$is shift invariant and locally finite-dimensional. Therefore, by Corollary 2.3 , there exists a constant $C$ independent of $h$ such that, for any $|\alpha| \leq m$,

$$
\left\|D^{\alpha} g(\cdot / h)\right\|_{p} \leq C h^{-|\alpha|}\|g(\cdot / h)\|_{p}, \quad \forall g \in S_{+} .
$$

Since $S_{0}(\psi)$ provides simultaneous approximation $k$, there exists $g_{h}$ in $S_{0}(\psi)$ such that

$$
\left\|D^{\alpha}\left(f-g_{h}(\cdot / h)\right)\right\|_{p} \leq B_{f} h^{k-|\alpha|}
$$


for all $|\alpha| \leq m$, where $B_{f}$ is some constant independent of $h$. As $g_{h}-s_{h}$ lies in $S_{+}$,

$$
\begin{aligned}
\left\|D^{\alpha}\left(f-s_{h}(\cdot / h)\right)\right\|_{p} & \leq\left\|D^{\alpha}\left(f-g_{h}(\cdot / h)\right)\right\|_{p}+\left\|D^{\alpha}\left(g_{h}(\cdot / h)-s_{h}(\cdot / h)\right)\right\|_{p} \\
& \leq B_{f} h^{k-|\alpha|}+C h^{-|\alpha|}\left\|g_{h}(\cdot / h)-s_{h}(\cdot / h)\right\|_{p} \\
& \leq B_{f} h^{k-|\alpha|}+C h^{-|\alpha|}\left(\left\|f-g_{h}(\cdot / h)\right\|_{p}+\left\|f-s_{h}(\cdot / h)\right\|_{p}\right) \\
& \leq\left(B_{f}+C B_{f}+C C_{f}\right) h^{k-|\alpha|} . \quad \square
\end{aligned}
$$

From the above proof we obtain the following

Corollary 3.2. Let $S \subset W_{p}^{m}\left(\mathbb{R}^{s}\right)$ be a shift-invariant subspace that is locally finite-dimensional. For any $f \in W_{p}^{m}\left(\mathbb{R}^{s}\right) \cap W_{p}^{k}\left(\mathbb{R}^{s}\right)$ and any $s_{h} \in S_{0}(\Phi)$, if $\left\|f-s_{h}(\cdot / h)\right\|_{p}=O\left(h^{k}\right)$, then $\left\|D^{\alpha}\left(f-s_{h}(\cdot / h)\right)\right\|_{p}=O\left(h^{k-|\alpha|}\right)$ for every $|\alpha| \leq m$.

This shows that any approximation scheme having approximation order $k$ in the $L_{p}\left(\mathbb{R}^{s}\right)$-norm automatically has simultaneous approximation order $(m, k)$ in this case.

Theorem 3.3. Let $S \subset L_{p}\left(\mathbb{R}^{s}\right) \cap L_{q}\left(\mathbb{R}^{s}\right)$ be a shift-invariant subspace with $1 \leq$ $p<q \leq \infty$, such that $S$ is locally finite-dimensional. If $S$ provides approximation order $k$ in the $L_{p}\left(\mathbb{R}^{s}\right)$-norm, then $S$ provides approximation order larger than or equal to $k-s(1 / p-1 / q)$ in the $L_{q}\left(\mathbb{R}^{s}\right)$-norm.

Proof. Let $\psi$ be a compactly supported function in $L_{\infty}\left(\mathbb{R}^{s}\right)$ such that $\psi$ satisfies the Strang-Fix conditions of order $k$. Then $S_{0}(\psi)$ provides approximation order $k$ in any $L_{r}\left(\mathbb{R}^{s}\right)$-norm for all $1 \leq r \leq \infty$. Moreover [1], there is a local approximation scheme

$$
Q_{h}: W_{r}^{k}\left(\mathbb{R}^{s}\right) \rightarrow S(\psi):=\psi^{* \prime} \ell_{r}\left(\mathbb{Z}^{s}\right)
$$

independent of $r$ such that $\left\|f-\left(Q_{h} f\right)(\cdot / h)\right\|_{r}=O\left(h^{k}\right)$.

Let $S_{+}$be the space spanned by $S$ and $S(\psi)$. It is clear that $S_{+}$is also shift invariant and locally finite-dimensional. By Corollary 2.4, there exists a constant $C$ such that $\|f\|_{q} \leq C\|f\|_{p}$, for all $f \in S_{+}$. For $f \in W_{p}^{k}\left(\mathbb{R}^{s}\right) \cap W_{q}^{k}\left(\mathbb{R}^{s}\right)$ and some $s_{h} \in S$,

$$
\begin{aligned}
\left\|f-s_{h}(\cdot / h)\right\|_{q} & \leq\left\|f-\left(Q_{h} f\right)(\cdot / h)\right\|_{q}+\left\|\left(Q_{h} f\right)(\cdot / h)-s_{h}(\cdot / h)\right\|_{q} \\
& =h^{s / q}\left\|Q_{h} f-s_{h}\right\|_{q}+O\left(h^{k}\right) \\
& \leq h^{s / q} C\left\|Q_{h} f-s_{h}\right\|_{p}+O\left(h^{k}\right) \\
& =h^{s / q-s / p} C\left\|\left(Q_{h} f\right)(\cdot / h)-s_{h}(\cdot / h)\right\|_{p}+O\left(h^{k}\right) \\
& \leq C h^{-s(1 / p-1 / q)}\left(\left\|f-\left(Q_{h} f\right)(\cdot / h)\right\|_{p}+\left\|f-s_{h}(\cdot / h)\right\|_{p}\right)+O\left(h^{k}\right) \\
& =O\left(h^{k-s(1 / p-1 / q)}\right)
\end{aligned}
$$

because $S$ provides approximation order $k$ in the $L_{p}\left(\mathbb{R}^{s}\right)$-norm.

When $s(1 / p-1 / q)<1, k$ is an integer, and $S$ provides an integral approximation order in the $L_{q}\left(\mathbb{R}^{s}\right)$-norm, it follows that $S$ also provides approximation order $k$ in the $L_{q}\left(\mathbb{R}^{s}\right)$-norm. In particular, if $S$ is known to provide an integral approximation order in the $L_{q}\left(\mathbb{R}^{s}\right)$-norm for all $q \geq p, S$ providing approximation order $k$ in the $L_{p}\left(\mathbb{R}^{s}\right)$-norm with $k$ an integer implies $S$ providing (at least) approximation 
order $k$ in the $L_{q}\left(\mathbb{R}^{s}\right)$-norm, for all $q \geq p$. As we shall see, in the univariate case, the approximation order of any shift-invariant space generated by a compactly supported function must be an integer, provided $p \geq 2$.

\section{Applichtion 2: Univariate approximation}

In the univariate case, there is an algebraic characterization for $S$ to provide approximation order $k$ even if $\hat{\varphi}(0)=0$ for every $\varphi \in S \cap L_{1}(\mathbb{R})$, where $S$ is a shift-invariant subspace of $L_{p}(\mathbb{R})$ that is locally finite-dimensional. Let $\Phi \subset$ $L_{p}(\mathbb{R})$ be a finite family of compactly supported functions. Recall that $S_{*}(\Phi)$ is the closure of $S_{0}(\Phi)$ in the topology of pointwise convergence. As proved by Jia [7], $S_{*}(\Phi)$ provides approximation order $k$ if and only if there is a compactly supported $\psi \in S_{*}(\Phi) \cap L_{p}(\mathbb{R})$ such that $\psi^{* \prime}$ maps $\Pi_{k-1}$ onto $\Pi_{k-1}$. Namely, $S_{*}(\Phi)$ provides approximation order $k$ if and only if there exist sequences $a_{\varphi}: \mathbb{Z} \rightarrow \mathbb{C}$ such that

$$
\psi=\sum_{\varphi \in \Phi} \varphi^{* \prime} a_{\varphi} \in L_{p}(\mathbb{R})
$$

is compactly supported and satisfies the Strang-Fix conditions of order $k$. This result reveals an intrinsic property of $S_{*}(\varphi)$ that provides a positive approximation order, as well as of $\varphi$ because $S_{*}(\varphi)$ is the closure of $S_{0}(\varphi)$ in the topology of pointwise convergence. As pointed out in [7], this follows that the approximation order provided by $S_{*}(\Phi)$ is an integer. Of another interest is that, when $\Phi \subset$ $L_{p}(\mathbb{R}) \cap L_{q}(\mathbb{R})$ with $p<q, S_{*}(\Phi)$ provides approximation order $k$ in the $L_{q}(\mathbb{R})$ norm if and only if it provides approximation order $k$ in the $L_{p}\left(\mathbb{R}^{s}\right)$-norm because, as we proved in Section $2, S_{*}(\Phi) \cap L_{p}\left(\mathbb{R}^{s}\right) \subset S_{*}(\Phi) \cap L_{q}\left(\mathbb{R}^{s}\right)$ and a compactly supported function in $L_{q}(\mathbb{R})$ lies in $L_{p}(\mathbb{R})$.

As $S_{*}(\Phi)$ is an infinite-dimensional space unless it is trivial, apparently it is nontrivial to determine if $S_{*}(\Phi)$ contains a compactly supported function that satisfies the Strang-Fix conditions of order $k$, even if $\Phi$ consists of a single compactly supported function in $L_{p}(\mathbb{R})$. For any given compactly supported function $\varphi \in L_{p}(\mathbb{R})$, it is more practically interesting to have a necessary and sufficient condition on the generator $\varphi$ itself for determining the approximation order provided by $S_{0}(\varphi)$. In the following we shall show that when $S=S_{0}(\varphi)$ with $\varphi \in L_{p}(\mathbb{R}) \cap L_{p^{\prime}}(\mathbb{R})$ compactly supported then $S_{*}(\varphi)$ and $S_{0}(\varphi)$ provide the same approximation order in the $L_{p}(\mathbb{R})$-norm for $p>1$. In particular, we prove that, for any compactly supported $\varphi \in L_{p}(\mathbb{R}) \cap L_{p^{\prime}}(\mathbb{R})$, with $p>1, S_{0}(\varphi)$ provides approximation order $k$ if and only if $\varphi=D^{m} \psi$ for some compactly supported $\psi \in W_{p}^{m}(\mathbb{R})$ that satisfies the Strang-Fix conditions of order $k+m$. In other words, $S_{0}(\varphi)$ provides approximation order $k$ if and only if there exists an integer $m \geq 0$ such that $D^{m} \hat{\varphi}(0) \neq 0$, and $D^{\alpha} \hat{\varphi}=0$ on $2 \pi \mathbb{Z} \backslash 0$ for all $0 \leq \alpha<k+m$.

Theorem 4.1. Let $1<p \leq \infty$ and $\varphi \in L_{p}(\mathbb{R}) \cap L_{p^{\prime}}(\mathbb{R})$ be compactly supported. Denote by $S_{p}(\varphi)$ and $S_{*}(\varphi)$ the closure of $S_{0}(\varphi)$ in the $L_{p}(\mathbb{R})$-norm and in the topology of pointwise convergence, respectively. Then $S_{0}(\varphi)$ provides approximation order $k$ in the $L_{p}(\mathbb{R})$-norm if and only if there exists a compactly supported function $\psi \in S_{2}(\varphi) \cap S_{p}(\varphi) \cap S_{*}(\varphi)$ such that $\psi$ satisfies the Strang-Fix conditions of order $k$.

Proof. We only need to prove the necessity because $S_{0}(\varphi)$ and $S_{p}(\varphi)$ provide the same approximation order. It is clear that if $S_{0}(\varphi)$ provides approximation order $k$ in the $L_{p}(\mathbb{R})$-norm, then so does $S_{*}(\varphi) \cap L_{p}(\mathbb{R})$. Therefore, $S_{*}(\varphi)$ contains a 
compactly supported function $\psi \in L_{p}(\mathbb{R})$ that satisfies the Strang-Fix conditions of order $k$. By Theorem 2.16 in [4], $\psi$ lies in $S_{2}(\varphi)$ if $p \geq 2$. By Corollary 2.5, $S_{2}(\varphi)$ is contained in $S_{p}(\varphi)$ when $p \geq 2$. In the case $p<2$, by Theorem 2.9 and Corollary $2.5, S_{p}(\varphi)=S_{*}(\varphi) \cap L_{p}(\mathbb{R}) \subset S_{2}(\varphi)$.

When $p \geq 2$, we have that $p^{\prime} \leq p$. Hence, if $p \geq 2$, then the condition that the compactly supported function $\varphi$ belongs to $L_{p^{\prime}}(\mathbb{R})$ is automatically satisfied. As an immediate consequence, we obtain that the approximation order provided by $S_{0}(\varphi)$ is an integer if $\varphi \in L_{p}(\mathbb{R}) \cap L_{p^{\prime}}(\mathbb{R})$ is compactly supported and $p>1$.

Corollary 4.2. Let $\varphi \in L_{p}(\mathbb{R}) \cap L_{p^{\prime}}(\mathbb{R})$ be a compactly supported function, with $1<p \leq \infty$. Then, $S_{0}(\varphi)$ provides approximation order $k$ in the $L_{p}(\mathbb{R})$-norm if and only if $S_{0}(\varphi)$ provides approximation order $k$ in the $L_{2}(\mathbb{R})$-norm.

Proof. It suffices to prove that $S_{0}(\varphi)$ providing approximation order $k$ in the $L_{2}(\mathbb{R})$ norm implies $S_{0}(\varphi)$ providing approximation order $\geq k$ in the $L_{p}(\mathbb{R})$-norm.

If $S_{2}(\varphi)$ provides approximation order $k$, then $S_{2}(\varphi)=S_{*} \cap L_{2}(\mathbb{R})$ contains a compactly supported function $\psi$ that satisfies the Strang-Fix conditions of order $k$. When $p>2$, from that $S_{2}(\varphi) \subset S_{p}(\varphi)$ it follows that $S_{p}(\varphi)$ also provides approximation order $k$. In the case $p<2$, we have that $\psi \in S_{*}(\varphi) \cap L_{p}\left(\mathbb{R}^{s}\right)=S_{p}(\varphi)$, because $\psi$ is compactly supported. So $S_{p}(\varphi)$ provides approximation order $k$.

As we know, for a nontrivial compactly supported $\varphi \in L_{2}(\mathbb{R}), S_{0}(\varphi)$ provides approximation order $k$ if and only if, for each $\alpha \in \mathbb{Z} \backslash 0$, there exist a neighborhood $\Omega_{\alpha}$ of the origin and a constant $C_{\alpha}$ such that (1.3) holds. So we have

Theorem 4.3. For any nontrivial compactly supported function $\varphi \in L_{p}(\mathbb{R}) \cap$ $L_{p^{\prime}}(\mathbb{R})$, with $p$ satisfying $1<p \leq \infty, S_{0}(\varphi)$ provides approximation order $k$ if and only if, for each $\alpha \in \mathbb{Z} \backslash 0$, there exist a neighborhood $\Omega_{\alpha}$ of the origin and a constant $C_{\alpha}$ such that

$$
|\hat{\varphi}(x+2 \pi \alpha)| \leq C_{\alpha}|x|^{k}|\hat{\varphi}(x)|, \quad \forall x \in \Omega_{\alpha} .
$$

For any nontrivial compactly supported function $\varphi \in L_{1}(\mathbb{R})$, there exists an integer $m \geq 0$ such that $D^{\alpha} \hat{\varphi}(0)=0$ for all nonnegative integers $\alpha<m$ but $D^{m} \hat{\varphi}(0) \neq 0$. As one can verify, (4.1) is equivalent to that $D^{\beta} \hat{\varphi}(2 \pi \alpha)=0$ for all integers $0 \leq \beta<k+m$, where $m$ is the smallest integer such that $D^{m} \hat{\varphi}(0) \neq 0$. When $m>0$, it is clear that

$$
\varphi_{1}(x):=\int_{-\infty}^{x} \varphi(t) d t
$$

is a compactly supported continuous function and for almost every $x \in \mathbb{R}$ we have that $D \varphi_{1}(x)=\varphi(x)$. Thus we obtain

$$
\widehat{\varphi_{1}}(x)=\frac{\hat{\varphi}(x)}{i x}, \quad \forall x \neq 0
$$

and $\widehat{\varphi_{1}}(0)=-i D \hat{\varphi}(0)$. When $m \geq 1$, define

$$
\varphi_{m}=\int_{-\infty}^{x} \frac{(x-t)^{m-1}}{(m-1) !} \varphi(t) d t .
$$

by induction we can prove that $\varphi_{m}$ is compactly supported, $D^{m} \varphi_{m}=\varphi$, and

$$
\widehat{\varphi_{m}}(x)=\frac{\hat{\varphi}(x)}{(i x)^{m}}, \quad \forall x \neq 0 .
$$


Note that $\lim _{x \rightarrow 0} \widehat{\varphi_{m}}(x)=(-i)^{m} D^{m} \hat{\varphi}(0) \neq 0$.

Corollary 4.4. Let $p>1, \varphi \in L_{p}(\mathbb{R}) \cap L_{p^{\prime}}(\mathbb{R})$ be a compactly supported function, and $m$ be the smallest integer such that $D^{m} \hat{\varphi}(0) \neq 0$. Then, $S_{0}(\varphi)$ provides approximation order $k$ in the $L_{p}(\mathbb{R})$-norm if and only if $\varphi=D^{m} \psi$ for some compactly supported function $\psi \in W_{p}^{m}(\mathbb{R})$ that satisfies the Strang-Fix conditions of order $k+m$.

Corollary 4.5. For $p>1$ and any compactly supported function $\varphi \in L_{p}(\mathbb{R}) \cap$ $L_{p^{\prime}}(\mathbb{R}), S_{0}(\varphi)$ provides approximation order $k \geq 1$ if and only if it locally contains $\Pi_{k-1}$.

Proof. We only need to prove the sufficiency because the necessity has been proved by Jia [8]. Since $S_{0}(\varphi)$ locally contains a nontrivial subspace $\Pi_{k-1}, \varphi$ is not trivial. So, $\varphi=D^{m} \psi$ for some compactly supported $\psi \in W_{p}^{m}(\mathbb{R})$ that satifies $\hat{\psi}(0) \neq 0$. Note that $D^{m} S_{0}(\psi)=S_{0}(\varphi)$. It follows that $S_{0}(\psi)$ locally contains $\Pi_{k+m-1}$. Since $\hat{\psi}(0) \neq 0$, we know that $S_{0}(\psi)$ locally contains $\Pi_{k+m-1}$ if and only if $\psi$ satisfies the Strang-Fix conditions of order $k+m$. Therefore, $S_{0}(\varphi)$ provides approximation order $k$.

Example. Let $\varphi$ be the function defined by (1.2). It is clear that $\varphi$ is bounded and is the first-order derivative of the following B-spline:

$$
\psi(x)= \begin{cases}x, & \text { if } 0 \leq x \leq 1 \\ 2-x, & \text { if } 1<x \leq 2 \\ 0, & \text { else }\end{cases}
$$

One can verify that $\psi$ satisfies the Strang-Fix conditions of order 2. Thus we know that $S_{0}(\varphi)$ does provide approximation order 1 in the $L_{p}(\mathbb{R})$-norm for $p>1$.

As we know, if $\hat{\varphi}(0)=0$, then $S_{0}(\varphi)$ cannot provide any positive approximation order in the $L_{1}(\mathbb{R})$-norm. When $\varphi \in L_{1}(\mathbb{R})$ is compactly supported and $\hat{\varphi}(0) \neq 0$, it is well known that $S_{0}(\varphi)$ provides approximation order $k>0$ if and only if $\varphi$ satisfies the Strang-Fix conditions of order $k$. So the approximation order provided by $S_{0}(\varphi)$ in the $L_{1}(\mathbb{R})$-norm is an integer.

\section{ACKNOWLEDGEMENTS}

This paper benefits much from communications with Dr. Rong-Qing Jia. The proof of Theorem 3.1 stemmed from the communications.

\section{REFERENCES}

1. C. de Boor, Quasiinterpolants and approximation power of multivariate splines, Computation of Curves and Surfaces (M. Gasca and C. Micchelli, eds.), Kluwer, Dordrecht, 1990, pp. 313345. MR 91i: 41009

2. __ Approximation order without quasi-interpolants, Approximation Theory (E. W. Cheney, C. K. Chui, and L. L. Schumaker, eds.), 1992, pp. 1-18. MR 94i:41042

3. C. de Boor and R. Devore, Partition of unity and approximation, Proc. Amer. Math. Soc. 98 (1985), 705-709. MR 86f:41003

4. C. de Boor, R. DeVore, and A. Ron, Approximation from shift-invariant subspaces of $L_{2}\left(\mathbb{R}^{s}\right)$, Trans. Amer. Math. Soc. 341 (1994), 787-806. MR 94d:41028

5. C. de Boor and G. Fix, Spline approximation by quasiinterpolants, J. Approx. Theory 8 (1973), 19-45. MR 49:5643

6. E. Cheney and W. Light, Quasi-interpolation with translates of a function having noncompact support, Constructive Approx. 1 (1992), 35-48. MR 93a:41027 
7. R. Jia, A characterization of the approximation order of translation invariant spaces of functions, Proc. Amer. Math. Soc. 111, (1991), 61-70. MR 91d:41018

8. - The Topelitz theorem and its applications to approximation theory and linear PDE's, Proc. Amer. Math. Soc. (to appear). CMP 94:13

9. R. Jia and J. Lei, Approximation by multiinteger translates of functions having non-compact support, J. Approx. Theory 72 (1993), 2-23. MR 94f:41024

10. W. Rudin, Functional analysis, McGraw-Hill, New York, 1973, pp. 90-91. MR 51:1315

11. I. Schoenberg, Contributions to the problem of approximation of equidistant data by analytic functions, Parts A \& B, Quart. Appl. Math. 4 (1946), 45-99, 112-141. MR 8:55d

12. G. Strang and G. Fix, A Fourier analysis of the finite element variation method, C.I.M.E.II (Ciclo 1971) (G. Geymonat, ed.), Constructive Aspects of Functional Analysis, 1973, pp. 793840.

13. K. Zhao, Simultaneous approximation from PSI spaces, J. Approx. Theory (to appear).

Department of Mathematics, University of Utah, Salt Lake City, Utah 84112

Current address: Structural Dynamics Research Corporation, 2000 Eastman Dr., Milford, Ohio 45150

E-mail address: kang.zhao@sdrc.com 